\title{
Satisfaction in motion: Subsequent search misses are more likely in moving search displays
}

\author{
Cary Stothart $^{1,2} \cdot$ Andrew Clement $^{1} \cdot$ James R. Brockmole $^{1}$
}

Published online: 8 May 2017

(C) Psychonomic Society, Inc. 2017

\begin{abstract}
People often conduct visual searches in which multiple targets are possible (e.g., medical X-rays can contain multiple abnormalities). In this type of search, observers are more likely to miss a second target after having found a first one (a subsequent search miss). Recent evidence has suggested that this effect may be due to a depletion of cognitive resources from tracking the identities and locations of found targets. Given that tracking moving objects is resource-demanding, would finding a moving target further increase the chances of missing a subsequent one? To address this question, we had participants search for one or more targets hidden among distractors. Subsequent search misses were more likely when the targets and distractors moved throughout the display than when they remained stationary. However, when the found targets were highlighted in a unique color, subsequent search misses were no more likely in moving displays. Together, these results suggest that the effect of movement is likely due to the increased cognitive demands of tracking moving targets. Overall, our findings reveal that activities that involve searching for moving targets (e.g., driving) are more susceptible to subsequent search misses than are those that involve searching for stationary targets (e.g., baggage screening).
\end{abstract}

Cary Stothart

cary.stothart.1@nd.edu

1 University of Notre Dame, Notre Dame, IN, USA

2 Department of Psychology, University of Notre Dame, 118 Haggar Hall, Notre Dame, IN 46556, USA
Keywords Attention - Visual search · Working memory · Object-based attention $\cdot$ Satisfaction of search $\cdot$ Multiple-target search

Visual search is a common activity, in both everyday and professional settings. In most visual search studies, participants must search for a single target among distractors. However, many real-world situations require people to search for multiple targets. For example, any bag that an airport security officer searches can contain multiple prohibited items. In this type of search, observers are more likely to miss a target if they have already found another one than in situations in which only one target is present. This effect, previously known as satisfaction of search, but more recently known as the subsequent search miss (SSM; Adamo, Cain, \& Mitroff, 2013) effect, has been extensively studied in the radiology literature (e.g., Tuddenham, 1962). For example, radiologists are more likely to miss a tumor when they have already found another abnormality in the same radiograph. In professions such as radiology and baggage screening, missing potentially dangerous targets can have severe consequences. Moreover, experience with a specific type of search does not prevent observers from making this type of error-both novice and professional searchers are susceptible to this effect (Biggs, Cain, Clark, Darling, \& Mitroff, 2013; Biggs \& Mitroff, 2014).

Researchers initially attributed SSM errors to observers prematurely ending a search after becoming "satisfied" with finding a target (Smith, 1967; Tuddenham, 1962). Indeed, these errors are more likely when observers spend a shorter amount of time searching for additional targets (Adamo, Cain, \& Mitroff, 2015a), although overall search times are usually unaffected by the detection of a first target (Berbaum et al., 1991; Berbaum, Franklin, Caldwell, \& Schartz, 2010; Fleck, Samei, 
\& Mitroff, 2010). More recently, SSM errors have been attributed to the depletion of cognitive resources associated with finding a first target (Berbaum et al., 1991; Cain \& Mitroff, 2013). For example, SSM errors may arise from the depletion of both attentional resources (SSM errors are correlated with failures of selective attention, such as the attentional blink, and are more common in crowded search displays; Adamo, Cain, \& Mitroff, 2013, 2015b, 2016) and working memory resources (SSM errors are more likely when the locations of found targets must be remembered; Cain \& Mitroff, 2013). In the latter case, simply removing found targets or highlighting them in a unique color greatly reduces the SSM effect.

Although nearly all studies of SSM errors involve static search displays, visual search in real-world situations is often more dynamic. For example, police officers must often search for multiple suspects in a crowd, while drivers must constantly scan the road for pedestrians and other hazards. If the resource depletion account is correct, these situations may be more susceptible to SSM errors than static search conditions. As a number of studies have indicated, tracking moving objects requires attention and working memory resources (Allen, McGeorge, Pearson, \& Milne, 2004, 2006; Tombu \& Seiffert, 2008). If SSM errors arise from tracking found targets, the added demands of tracking moving targets should deplete additional cognitive resources, resulting in a greater likelihood of missing subsequent targets. This outcome would not only support the key predictions of the resource depletion account, but would also have important consequences for many real-world activities. For example, if dynamic search conditions are more susceptible to SSM errors, preventing these errors could reduce the number of causalities in everyday activities such as driving. On the other hand, if tracking moving targets does not increase the likelihood of SSM errors, this would suggest that these errors do not arise from the depletion of cognitive resources. Instead, this outcome would be consistent with other theoretical accounts, which assume that SSM errors are largely driven by perceptual differences among the targets (Berbaum et al., 1991; Berbaum et al., 1990).

In two experiments, we assessed whether target movement influences the likelihood of observing SSM errors. In Experiment 1, we compared SSM errors in moving displays, which contained both moving targets and distractors, to stationary displays, which contained both stationary targets and distractors. In Experiment 2, we highlighted found targets in a unique color, thereby reducing the difficulty of tracking them. This allowed us to assess whether any differences in the rate of SSM errors were due to the added demands of tracking found targets.

\section{Experiment 1}

If tracking found targets depletes cognitive resources, the added demands of tracking moving targets should deplete additional resources, resulting in an increased rate of SSM errors (Berbaum et al., 1991; Cain \& Mitroff, 2013). In Experiment 1, we tested this hypothesis by having participants search moving or stationary displays. If SSM errors arise from the depletion of cognitive resources, these errors should be more likely to occur in moving search displays.

\section{Method}

The materials, analyses, and data from Experiment 1 are available on the Open Science Framework (https://osf.io/srvja/).

Participants Participants were from the United States and were recruited and tested online using Amazon Mechanical Turk (MTurk). To participate, participants needed an MTurk approval rating of at least $95 \%$ and a device with a keyboard. Data were collected from a total of 105 participants ${ }^{1}$; however, data from 11 participants were excluded for one or more of the reasons listed in Table 1. Of the remaining 94 participants, 42 searched moving displays ( 28 females, 14 males; median age = 31.50 years, $I Q R=9.48)$, and 52 searched stationary displays (40 females, 12 males; median age $=38.50$ years, $I Q R=12.53$ ).

Materials and procedure Participants completed the experiment on their own computers. The visual search task was programmed in JavaScript, and the surveys were programmed in HTML/CSS.

Participants first completed a visual search task that was based on the task used by Fleck et al. (2010; see Fig. 1). In this task, participants viewed displays of 20 black objects on a white background $(726 \times 546$ pixels). Of these 20 objects, one, two, or three were upright perfect black Ts (targets), while the remaining 19,18 , or 17 were upright imperfect black Ts (distractors). ${ }^{2}$ Imperfect Ts were created by offsetting the vertical bar of the T 15,12, 9, or 6 pixels to the left or right of center. Each item subtended $37 \times 37$ pixels. In the moving displays, these objects moved along linear trajectories at 60 pixels/s, bouncing off the edges of the display and each other when they came within a distance of 19 pixels. In the stationary displays, the objects did not move, but were spaced at least 19 pixels away from each other. Participants searched for and clicked on perfect Ts (any clicked object turned yellow for $200 \mathrm{~ms}$ before turning back to black). Each trial lasted for a maximum of $15 \mathrm{~s}$, although participants could end a trial early by pressing a "Done" button located below the display. If participants ran out of time, they were shown a message that

\footnotetext{
${ }^{1}$ No previous study has assessed the effect of target movement on SSM errors; thus, we did not know what size of effect to expect. Therefore, we aimed for a sample size of 100 , which gave us an $80 \%$ chance of detecting a movement effect as small as $10 \%$ (i.e., the difference in the rates of SSM errors between movement conditions).

${ }^{2}$ The three-target trials were added to ensure that participants would continue searching after finding two targets, but these trials were not of primary interest for our analyses.
} 
Table 1 Numbers and percentages of participants failing each exclusion criterion in the study

\begin{tabular}{|c|c|c|c|}
\hline Exclusion rule & Exp. 1 & Exp. 2 & All Exp. \\
\hline Already participated in the experiment & $0(0 \%)$ & $6(1.65 \%)$ & $6(1.28 \%)$ \\
\hline Reported not having normal or corrected-to-normal vision & $4(3.81 \%)$ & $11(3.00 \%)$ & $15(3.21 \%)$ \\
\hline Failed to pay attention to the instructions & $3(2.86 \%)$ & $8(2.20 \%)$ & $11(2.35 \%)$ \\
\hline $\begin{array}{l}\text { Entered a nonsensical response for one of the open response } \\
\text { questions or reported that the task did not work correctly }\end{array}$ & $3(2.86 \%)$ & $11(3.00 \%)$ & $14(2.99 \%)$ \\
\hline Median search time below $1,000 \mathrm{~ms}$ & $1(0.95 \%)$ & $2(0.05 \%)$ & $3(0.64 \%)$ \\
\hline Total tested & 105 & 363 & 468 \\
\hline Total excluded & 11 & 38 & 49 \\
\hline
\end{tabular}

Participants could be excluded for multiple reasons

encouraged them to search faster on future trials. Participants completed a total of 70 trials, with the first ten trials being practice with feedback (number of perfect Ts found out of the total number in the display, and the false positive rate). Participants received no feedback on their performance for the remaining 60 trials. Ten percent of trials contained three perfect Ts, $30 \%$ contained two perfect Ts, and the remaining $60 \%$ of trials contained only one perfect $\mathrm{T}$. We chose this target distribution to bias participants' expectations for a low number of targets. Similar distributions have produced pronounced SSM effects in previous studies (e.g., Fleck et al., 2010). The trials were randomly shuffled for each participant. The ten practice trials always had the same target distribution.

After completing the visual search task, participants completed a survey about the quality of the task, the quality of their vision, and basic demographic information. We also tested whether participants were paying attention to our instructions. On one screen, we asked participants to select the middle item in a list of numbers and remember it for a later test. On the next screen, we asked them to enter the number they had selected on the previous screen. Participants failed this test if they selected an incorrect number on the first screen or entered an incorrect number on the second screen; these participants were excluded from our analyses.

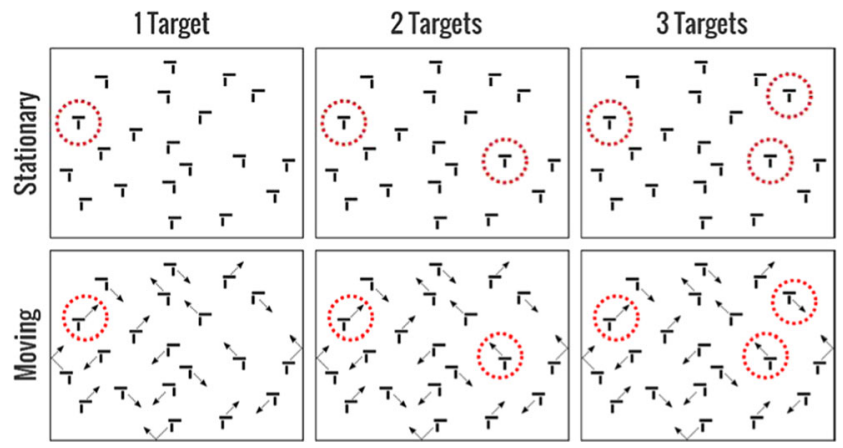

Fig. 1 The visual search task. Experiment 1 trials included up to three targets, and Experiment 2 trials included up to two targets. Targets are circled in red (the circles were not shown in either experiment)

\section{Results}

All analyses were conducted in $\mathrm{R}$ version 3.3.1 ( $\mathrm{R}$ Development Core Team, 2016). Before analyzing the data, we removed trials on which participants never clicked the "Done" button (3.09\% of all trials), completed the trial in under $500 \mathrm{~ms}(0.62 \%)$, or clicked on a distractor or alreadyclicked target $(6.54 \%)$. The percentages of acceptable trials for each target condition in which zero, one, or two targets were found are provided in Table 2. For each participant, we calculated accuracy in detecting the remaining target for each target condition by dividing the number of trials on which participants found all targets by the total number of trials on which the participant found at least $X-1$ targets, with $X$ being the number of targets in the trial (see Fig. 2a). We then submitted these accuracies to a linear mixed-effects model with Display Type (moving vs. stationary) and Number of Targets in a Trial (1 vs. 2) entered as factors. Participant ID was used as a random intercept. Because we were only interested in assessing whether SSM errors were affected by target movement, we only report the interactions from this analysis. Information about our main effects is available at https://osf.io/srvja/.

The purpose of Experiment 1 was to determine whether the rate of SSM errors is greater in moving than in stationary search displays. Our results suggest that this was the case. The difference in mean accuracy between one- and twotarget trials was 13.25 percentage points larger in moving than in stationary displays (see Fig. $2 \mathrm{~b}$ ), $F(1,92)=10.66, p=.002$ (interaction between display type and number of targets: 1 or 2). ${ }^{3}$ Importantly, this effect was not due to differences in the time available to find the remaining target; participants were

\footnotetext{
${ }^{3}$ For archival purposes, we note that the mean accuracies for the three-target filler trials were a little lower than those for the two-target trials: 55.39\% [95\% CI: $47.92 \%, 62.87 \%]$ and $35.89 \%$ [27.57\%, 44.22\%] for stationary and dynamic three-target displays, respectively. The one- versus three-target $(15.57 \%), F(1,90)=6.44, p=.013$, but not the two- versus three-target $(2.31 \%), F(1,90)=0.12, p=.731$, mean accuracy difference was greater in moving than in stationary displays.
} 
Table 2 Percentages of trials in each target condition of Experiment 1 on which zero, one, or two targets were found

\begin{tabular}{|c|c|c|c|c|c|}
\hline \multirow[t]{2}{*}{ Display type } & \multirow[t]{2}{*}{$N$ Targets } & \multicolumn{3}{|c|}{$\%$ of trials in each target condition on which participants found: } & \multirow[t]{2}{*}{$N$ Trials } \\
\hline & & No targets & One target & Two targets & \\
\hline Stationary & 1 & $6.59 \%$ & $93.41 \%$ & N/A & 1,761 \\
\hline Stationary & 2 & $1.80 \%$ & $31.91 \%$ & $66.29 \%$ & 890 \\
\hline Moving & 1 & $9.92 \%$ & $90.08 \%$ & N/A & 1,270 \\
\hline Moving & 2 & $3.27 \%$ & $49.92 \%$ & $46.81 \%$ & 643 \\
\hline
\end{tabular}

no slower at finding the first target in moving $(M=3,267 \mathrm{~ms}$, $S D=733.23)$ than in stationary $(M=3,361.51 \mathrm{~ms}, S D=$ 882.62 ) displays, $t(92)=0.56, p=.580$.

\section{Discussion}

As the results of Experiment 1 indicate, SSM errors were more likely when observers searched for moving rather than stationary targets. While participants were more likely to miss a subsequent target after finding another target, this effect was greater when they searched moving rather than stationary displays.

\section{Experiment 2}

According to the resource depletion account, any effect of movement on the rate of SSM errors should be due to the additional cognitive demands of tracking moving targets (Berbaum et al., 1991; Cain \& Mitroff, 2013). In Experiment 2, we tested this hypothesis by highlighting found targets in a unique color, thereby reducing the cognitive resources required to track them (Cain \& Mitroff, 2013; Feria, 2012; Makovski \& Jiang, 2009). If the effect of target movement on SSM errors is due to the added demands of tracking moving targets, highlighting the found targets should diminish this effect.
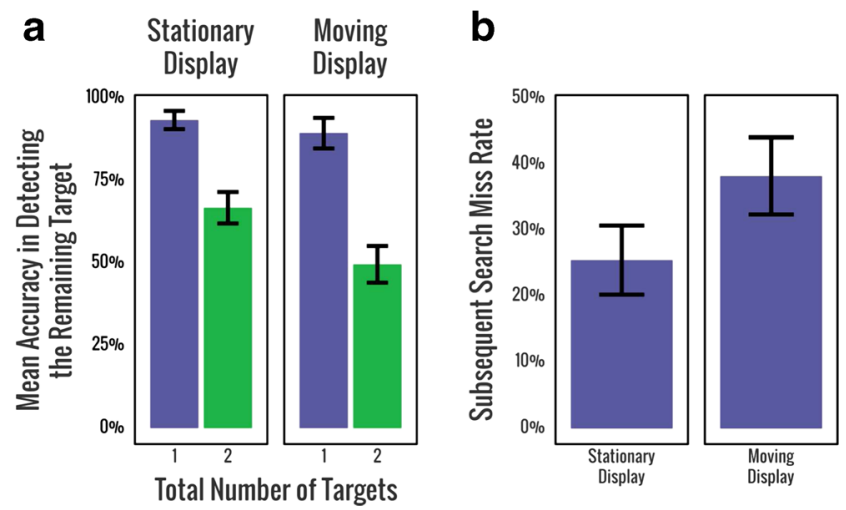

Fig. 2 (a) Mean accuracy for each condition. (b) The SSM rate for each condition (one-target trial mean accuracy - two-target trial mean accuracy). Error bars represent between-participants $95 \%$ confidence intervals in both plots

\section{Method}

We preregistered our testing plan, stopping rule, and exclusion criteria for Experiment 2 on the Open Science Framework (https:/osf.io/srvja/). The materials, analyses, and data for this experiment are also available on this site.

Participants Data were collected from a total of 363 participants on MTurk using the same requirements as in Experiment $1^{4}$; however, the data from 38 participants were removed for one or more of the reasons listed in Table 1. Of the remaining 325 participants, 87 searched moving displays in which found targets were not highlighted (53 females, 34 males; median age $=40$ years, $I Q R=23)$, 75 searched stationary displays in which found targets were not highlighted (39 females, 36 males; median age $=36$ years, $I Q R=18.5), 77$ searched moving displays in which found targets were highlighted (40 females, 37 males; median age $=39$ years, $I Q R=23$ ), and 86 searched stationary displays in which found targets were highlighted (55 females, 31 males; median age $=35$ years, $I Q R=18$ ).

Materials and procedure The visual search task and postexperiment surveys were identical to those in Experiment 1, with the following exceptions. First, to increase the number of one- and two-target trials, and to make our experiment more consistent with previous SSM experiments, we did not include any three-target trials (30\% of trials contained two targets, and $70 \%$ of trials contained one target). Second, participants were also assigned to one of two conditions. In the highlight condition, any clicked targets turned green for the remainder of the trial, while any clicked distractors turned red for $200 \mathrm{~ms}$ before turning back to black. In the no-highlight condition, any clicked targets turned green for $200 \mathrm{~ms}$ before turning back to black, while any clicked distractors turned red for $200 \mathrm{~ms}$ before turning back to black. The reason for temporarily changing the color of found

\footnotetext{
${ }^{4}$ We chose to collect data on a minimum of 300 usable participants, because this sample size would give us at least an $80 \%$ chance of detecting a population threeway interaction between movement condition (moving vs. stationary), number of found targets ( 0 vs. 1 ), and highlight condition (highlight vs. no - highlight). This assumes that the size of the population two-way interaction between movement and number of found targets in the no-highlight condition was the same as that for the two-way interaction observed in Experiment 1. It also assumes that there was no population two-way interaction in the highlight condition.
} 
targets in the no-highlight condition and distractors in both conditions was to keep feedback consistent across the conditions.

\section{Results}

All analyses were conducted in R version 3.3.1 (R Development Core Team, 2016). Before analyzing the data, we again removed trials on which participants never clicked the "Done" button ( $8.86 \%$ of all trials), completed the trial in under $500 \mathrm{~ms}$ $(0.01 \%)$, or clicked on a distractor or already-found target (7.37\%). The percentages of acceptable trials for each target condition on which zero, one, or two targets were found are provided in Table 3. We calculated accuracy in the same way as in Experiment 1 (see Fig. 3a). Aside from the added factor of Highlight Condition, the analysis in this experiment was the same as that in Experiment 1.

The purpose of Experiment 2 was to determine whether highlighting clicked targets (and thus making object tracking unnecessary) would reduce the effect of target movement on the SSM effect. The results of our analysis suggest that this was the case. When clicked targets were not highlighted, the difference in the rates of SSM errors for moving and stationary displays was $10.08 \%$ (cf. $13.25 \%$ in Exp. 1; see Fig. 3b), $F(1$, $153)=10.13, p=.002$. When clicked targets were highlighted, however, this difference was only $1.13 \%, F(1,158)=0.09, p=$ .764; three-way interaction: $F(1,311)=3.34, p=.069$. As in Experiment 1, the movement effect did not depend on the amount of time available to find the remaining target, since participants were no slower at finding the first target in moving $(M=$ $3,474.67 \mathrm{~ms}, S D=912.61)$ than in stationary $(M=3,508.37, S D$ $=848.12$ ) displays, $t(317)=0.34, p=.733$.

\section{Discussion}

By highlighting found targets in a unique color and thereby reducing the amount of cognitive resources required to track them, we were able to substantially reduce the effect of target movement on SSM errors. This finding provides support for
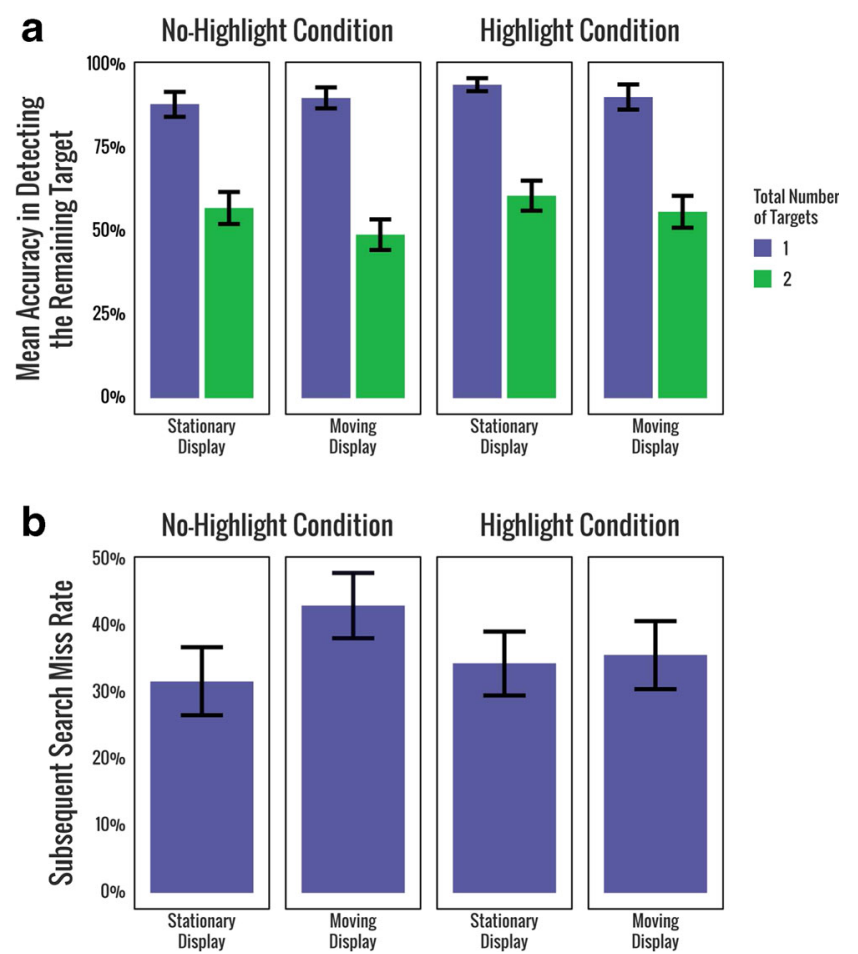

Fig. 3 (a) Mean accuracy for each condition. (b) The SSM rate for each condition (one-target trial mean accuracy - two-target trial mean accuracy). Error bars represent between-participants $95 \%$ confidence intervals in both plots

the resource depletion account and suggests that the effect of target movement on SSM errors is due to the added demands of tracking moving targets.

\section{General discussion}

When searching displays that contain multiple targets, observers are more likely to miss a subsequent target after finding a first one. This type of error, known as either satisfaction of search or $S S M$, is pervasive in many real-world situations. Here we replicated this effect under both static and dynamic

Table 3 Percentages of trials in each target condition in Experiment 2 on which zero, one, or two targets were found

\begin{tabular}{|c|c|c|c|c|c|c|}
\hline \multirow[t]{2}{*}{ Highlight condition } & \multirow[t]{2}{*}{ Display type } & \multirow[t]{2}{*}{$N$ Targets } & \multicolumn{3}{|c|}{$\%$ of trials in each target condition on which participants found: } & \multirow[t]{2}{*}{$N$ Trials } \\
\hline & & & No targets & One target & Two targets & \\
\hline No-Highlight & Stationary & 1 & $9.56 \%$ & $90.44 \%$ & N/A & 3,033 \\
\hline No-Highlight & Stationary & 2 & $3.16 \%$ & $38.62 \%$ & $58.22 \%$ & 1,362 \\
\hline No-Highlight & Moving & 1 & $8.40 \%$ & $91.60 \%$ & N/A & 2,713 \\
\hline No-Highlight & Moving & 2 & $3.09 \%$ & $48.87 \%$ & $48.04 \%$ & 1,199 \\
\hline Highlight & Stationary & 1 & $4.00 \%$ & $96.00 \%$ & N/A & 3,298 \\
\hline Highlight & Stationary & 2 & $1.68 \%$ & $36.88 \%$ & $61.44 \%$ & 1,486 \\
\hline Highlight & Moving & 1 & $6.93 \%$ & $93.07 \%$ & N/A & 2,685 \\
\hline Highlight & Moving & 2 & $1.93 \%$ & $42.11 \%$ & $55.96 \%$ & 1,242 \\
\hline
\end{tabular}


search conditions. When searching for a small number of targets among distractors, participants were more likely to miss a target if they had already found another one in the display. More importantly, this effect was greater for moving than for stationary displays. Based on the results of our experiments, this increase in the rate of SSM errors was likely due to the added demands of tracking moving as compared to stationary targets.

Overall, our results support key predictions of the resource depletion account. According to this account, any manipulation that increases the demands of tracking found targets should increase the rate of SSM errors. Conversely, any manipulation that decreases these demands should decrease the rate of SSM errors (Cain \& Mitroff, 2013; McCarley et al., 2006). Here we found an increased likelihood of SSM errors when participants searched moving rather than stationary displays. This result is consistent with the resource depletion account, because tracking moving targets requires attention and working memory resources (Allen et al., 2004, 2006; Tombu \& Seiffert, 2008). However, when the found targets were highlighted, the likelihood of SSM errors were comparable for moving and stationary displays. Thus, reducing the demands of tracking found targets decreased the effect of movement. It is worth noting that this manipulation did not fully eliminate SSM errors - the rate of these errors was still approximately $30 \%$ when found targets were highlighted.

Importantly, our results also suggest that activities that involve tracking moving objects may be more susceptible to SSM errors. This is concerning, because many dangerous real-world activities involve searching for moving targets under cognitive load. For example, drivers must scan the road for pedestrians, animals, and approaching vehicles, all while controlling their own vehicle. Drivers may also search for these targets while conducting ill-advised activities such as talking or texting on a cell phone-activities that further reduce the probability of spotting a hazard (Strayer, Drews, \& Johnston, 2003). Recent evidence has shown that SSM errors are possible when traffic is at a standstill (Sall \& Feng, 2016). Our results suggest that these errors are even more likely when traffic is moving. Future research should take this finding into consideration, since traffic accidents account for large numbers of casualties in both the United States and other countries (World Health Organization, 2015).

In summary, we found that searching moving search displays increases the likelihood of observing SSM errors. Based on our results, this effect is likely due to the additional cognitive resources required to track moving targets. Together, these findings provide further evidence for the resource depletion account, which suggests that SSM errors arise partly from tracking the identities and locations of previously found targets. Our findings also have implications for everyday activities that involve searching for moving targets, such as driving.

\section{References}

Adamo, S. H., Cain, M. S., \& Mitroff, S. R. (2013). Self-induced attentional blink: A cause of errors in multiple-target visual search. Psychological Science, 24, 2569-2574. doi:10.1177/ 0956797613497970

Adamo, S. H., Cain, M. S., \& Mitroff, S. R. (2015a). Satisfaction at last: Evidence for the "satisfaction" hypothesis for multipletarget search errors. Visual Cognition, 23, 821-825. doi:10. 1080/13506285.2015.1093248

Adamo, S. H., Cain, M. S., \& Mitroff, S. R. (2015b). Targets need their own personal space: Effects of clutter on multiple-target search accuracy. Perception, 44, 1203-1214. doi:10.1177/0301006615594921

Adamo, S. H., Cain, M. S., \& Mitroff, S. R. (2016). An individual differences approach to multiple-target visual search errors: How search errors relate to different characteristics of attention. Vision Research. doi:10.1016/j. visres.2016.10.010.

Allen, R., McGeorge, P., Pearson, D., \& Milne, A. (2004). Attention and expertise in multiple target tracking. Applied Cognitive Psychology, 18, 337-347. doi:10.1002/acp.975

Allen, R., McGeorge, P., Pearson, D., \& Milne, A. (2006). Multiple-target tracking: A role for working memory? Quarterly Journal of Experimental Psychology, 59, 1101-1116. doi:10.1080/ 02724980543000097

Berbaum, K. S., Franken, E. A., Jr., Dorfman, D. D., Rooholamini, S. A., Coffman, C. E., Conell, S. H., ... Kao, S. C. (1991). Time course of satisfaction of search. Investigative Radiology, 26, 640-648.

Berbaum, K. S., Franken, E. A., Jr., Dorfman, D. D., Rooholamini, S. A., Kathol, M. H., Barloon, T. J., ... Montgomery, W. J. (1990). Satisfaction of search in diagnostic radiology. Investigative Radiology, 25, 133-140.

Berbaum, K. S., Franklin, E. A., Jr., Caldwell, R. T., \& Schartz, K. M. (2010). Satisfaction of search in traditional radiographic imaging. In E. Samei \& E. Krupinski (Eds.), The handbook of medical image perception and techniques (pp. 107-138). Cambridge, UK: Cambridge University Press.

Biggs, A. T., Cain, M. S., Clark, K., Darling, E. F., \& Mitroff, S. R. (2013). Assessing visual search performance differences between Transportation Security Administration Officers and nonprofessional searchers. Visual Cognition, 21, 330-352. doi:10.1080/13506285.2013.790329

Biggs, A. T., \& Mitroff, S. R. (2014). Different predictors of multipletarget search accuracy between non-professional and professional visual searchers. Quarterly Journal of Experimental Psychology, 67, 1335-1348. doi:10.1080/17470218.2013.859715

Cain, M. S., \& Mitroff, S. R. (2013). Memory for found targets interferes with subsequent performance in multiple-target visual search. Journal of Experimental Psychology: Human Perception and Performance, 39, 1398-1408. doi:10.1037/a0030726

Feria, C. S. (2012). The effects of distractors in multiple object tracking are modulated by the similarity of distractor and target features. Perception, 41, 287-304. doi:10.1068/p7053

Fleck, M. S., Samei, E., \& Mitroff, S. R. (2010). Generalized "satisfaction of search": Adverse influences on dual-target search accuracy. Journal of Experimental Psychology: Applied, 16, 60-70. doi:10.1037/a0018629

Makovski, T., \& Jiang, Y. V. (2009). The role of visual working memory in attentive tracking of unique objects. Journal of Experimental Psychology: Human Perception and Performance, 35, 1687-1697. doi:10.1037/a0016453

McCarley, J. S., Kramer, A. F., Boot, W. R., Peterson, M. S., Wang, R. F., \& Irwin, D. E. (2006). Oculomotor behavior in visual search for multiple targets. Visual Cognition, 14, 685-703. doi:10.1080/ 13506280500194147

R Development Core Team. (2016). R: A language and environment for statistical computing (Version 3.3.1) [Computer software]. Vienna, 
Austria: R Foundation for Statistical Computing. Available from www.R-project.org

Sall, R. J., \& Feng, J. (2016). Better off alone: The presence of one hazard impedes detection of another in simulated traffic scenes. Proceedings of the Human Factors and Ergonomics Society Annual Meeting, 60, 1444-1448. doi:10.1177/1541931213601331

Smith, M. J. (1967). Error and variation in diagnostic radiology. Springfield, IL: C. C. Thomas.

Strayer, D. L., Drews, F. A., \& Johnston, W. A. (2003). Cell phoneinduced failures of visual attention during simulated driving.
Journal of Experimental Psychology: Applied, 9, 23-32. doi:10. 1037/1076-898X.9.1.23

Tombu, M., \& Seiffert, A. E. (2008). Attentional costs in multipleobject tracking. Cognition, 108, 1-25. doi:10.1016/j.cognition. 2007.12.014

Tuddenham, W. J. (1962). Visual search, image organization, and reader error in roentgen diagnosis. Radiology, 78, 694-704.

World Health Organization. (2015). Global status report on road safety 2015. Retrieved from www.who.int/violence_injury_prevention/ road_safety_status/2015/en/ 